Conclusions: SMP facilitates early diagnosis of allopurinol allergy/DiHS which occurred during the first $2-6$ weeks after initiation. Whether early cessation of allopurinol prevents development of SCAR, and reduces the need for HLA-B*5801 testing will require a larger prospective study.

References:

[1] Wang CW, Dao RL, Chung WH. Immunopathogenesis and risk factors for allopurinol severe cutaneous adverse reactions. Curr Opin Allergy Clin Immunol 2016;16:339-45.

[2] Dong D, Tan-Koi WC, Teng GG, Finkelstein E, Sung C. Cost-effectiveness analysis of genotyping for HLA-B*5801 and an enhanced safety program in gout patients starting allopurinol in Singapore. Pharmacogenomics 2015;16:178193.

Disclosure of Interest: None declared

DOI: 10.1136/annrheumdis-2017-eular.2531

\section{THU0416 RAPID TOPHUS RESOLUTION IN CHRONIC REFRACTORY GOUT PATIENTS TREATED WITH PEGLOTICASE}

B. Mandell ${ }^{1}$, A. Yeo ${ }^{2}$, P. Lipsky ${ }^{3} .{ }^{1}$ Department of Medicine, Cleveland Clinic, Cleveland, OH; ${ }^{2}$ Horizon Pharma, Lake Forest, IL; ${ }^{3}$ AMPEL BioSolutions, LLC, Charlottesville, VA, United States

Background: It has been suggested that the velocity of resolution of tophi in persons with chronic tophaceous gout is related to the serum urate levels. ${ }^{1}$ However, few subjects with a persistent serum urate less than $4.0 \mathrm{mg} / \mathrm{dL}$ were studied. Pegloticase is a recombinant uricase conjugated to polyethylene glycol approved in the US for treatment of patients with chronic refractory gout. It profoundly decreases serum uric acid in responders to $<1 \mathrm{mg} / \mathrm{dL}$. The results from the pegloticase clinical trials provided the opportunity to determine the impact of persistent and markedly low levels of serum urate on the velocity of tophus resolution.

Objectives: To assess the velocity of tophus resolution in subjects treated with pegloticase for chronic refractory gout.

Methods: This analysis used results from two randomized controlled trials (RCTs) of 6 months duration. 2,3 For tophus measurements, serial standardized digital photographs were analyzed by a blinded reader using computer-assisted quantitative measurement software. Subjects were defined as responders and nonresponders based upon maintenance of a serum urate $<6 \mathrm{mg} / \mathrm{dL}$ during intensive monitoring periods after 3 and 6 months of treatment.

Results: During the 6 months of the RCTs, a total of 952 tophus measurements were analyzed in 87 subjects, including 341 in 30 responders; 361 in 36 nonresponders receiving pegloticase infusions; and 250 in 21 subjects receiving placebo infusions. Mean serum urate levels in these subjects were $10.1,0.3$ and $0.3 \mathrm{mg} / \mathrm{dL}$ at baseline, 3 months and 6 months in responders; $10.7,8.9$ and 9.6 $\mathrm{mg} / \mathrm{dL}$ in nonresponders; and 10.2, 9.8 and $9.7 \mathrm{mg} / \mathrm{dL}$ in placebo treated patients, respectively. At baseline, the mean tophus area in responders was $581.6+$ $742.7 \mathrm{~mm}^{2}$ (mean $\pm \mathrm{SD} ; \mathrm{n}=90$ tophi); in nonresponders it was $676.5+/-1416.6$ $\mathrm{mm}^{2}$ ( $\mathrm{n}=93$ tophi); and in placebo treated subjects it was $672.9+/-1039.5 \mathrm{~mm}^{2}$ $(n=66$ tophi). By regression analysis, the velocity of tophus resolution over the 6 months of treatment was $50.1 \mathrm{~mm}^{2} /$ month in responders; $14.0 \mathrm{~mm}^{2} / \mathrm{month}$ in nonresponders; and $13.9 \mathrm{~mm}^{2} /$ month in placebo treated patients (responders versus nonresponders or responders vs placebo treated subjects $(p=0.001))$. In responders, the mean time to total tophus resolution was estimated to be 347 days (11.5 months, with a range of 5.6-36.4 months). During the 6 month treatment period, the area under the curve (AUC) of multiple serum urate measurements in responders was $6,067.9+/-6,781.6 \mathrm{mg} / \mathrm{dL} \mathrm{hr}$ compared with $34,647.4+/-8,586.7$ and $42,451.1+/-6,396.1 \mathrm{mg} / \mathrm{dL} \cdot \mathrm{hr}$ in nonresponders and placebo treated subjects, respectively $(p<0.001)$. In responders, there was a significant correlation between the velocity of tophus resolution and serum urate AUC $(p=0.009)$.

Conclusions: Pegloticase treatment causes a rapid resolution of tophi in responders as predicted from the profound and persistent serum urate lowering associated with this therapy.

References:

[1] Perez-Ruiz F et al, Arthritis Rheum 2002; 47:356-60.

[2] Sundy JS, et al. JAMA. 2011;306:711-720.

[3] Becker MA, et al. Ann Rheum Dis. 2013;72:1469-1474.

Disclosure of Interest: B. Mandell Grant/research support from: Horizon, Consultant for: Horizon, Ironwood, A. Yeo Consultant for: Horizon Pharma, P. Lipsky Consultant for: AstraZeneca, Celgene, EMD Serono, GSK, Horizon Pharma, Janssen, Medimmune, Pfizer, Roche, Sanofi, UCB

DOI: 10.1136/annrheumdis-2017-eular.5417

\section{THU0417 PERIPHERAL NEUROPATHY IN PATIENTS WITH GOUT. ALTERATIONS BEYOND LOCAL DAMAGE}

C.O. Lopez Lopez ${ }^{1}$, E. Corzo Dominguez ${ }^{1}$, M.D.L.L. Montes-Castillo ${ }^{1}$ H. Llinas ${ }^{2}$, E. Alvarez Hernandez ${ }^{2}$, I. Peláez Ballestas ${ }^{2}$, R. Burgos Vargas ${ }^{2}$, J. Vazquez-Mellado ${ }^{2}{ }^{1}$ Rehabilitation; ${ }^{2}$ Rheumatology, Hospital General de Mexico, Mexico city, Mexico

Background: Peripheral neuropathies (PN), are peripheral nervous system disorders associated to several causes. According to distribution are classified as: Local (mononeuropathy [MNP]) or Systemic (Multiple mononeuropathy [MNPM] and polyneuropathy [PNP]). PN in gout has been scarcely described. Previous reports only consider MNP of median nerve at the wrist and MNP of the ulnar nerve at the elbow, due to tophus compression.

Objectives: To describe the frequency and characteristics of $\mathrm{PN}$ in patients with gout and its association to gout related variables, co-morbidity and treatment.

Methods: Consecutive patients from GRESGO, a cohort of 450 gout (ARA/CGD/ACR-EULAR) patients seen for the first time at Rheumatology department and treated according to published guidelines for gout. Variables included: demographic, clinical, biochemical data, $\mathrm{HAQ}$ and 3 questionnaires for PN (DN4, LANSS and MNSI) previously translated and validated in our country. We performed Nerve Conduction Studies (NCS) following AAME guidelines (Include:Sensory action potential [sural, ulnar and median nerves], Compound muscle action potential [peroneal, tibial, median and ulnar nerves] and late F-wave [tibial and ulnar nerves]). This protocol was approved by the local IRB and the patients signed an informed consent. Statistical analysis: Student's t test, Mann-Whitney $\mathrm{U}$ test and $\mathrm{X} 2$.

Results: We included 162 gout patients, $98 \%$ males, $72 \%$ tophaceous gout, $48 \%$ severe tophaceous gout (STG), mean age $49.4 \pm 12$ years, $14 \pm 10$ years of disease duration, educational level $8 \pm 4$ years, BMI $27.9 \pm 4.6 \mathrm{~kg} / \mathrm{m}^{2}$.

According to questionnaires: $56 \%$ DN4, $45 \%$ MNSI and $36 \%$ LANSS could be classified as PN. Sixty five percent had abnormal NCS: MNP: $52 \%$, most of them (58\%) neuropraxia. PNP $35 \%$ and $13 \%$ MNPM in them, axonal damage was reported in $88 \%$. MNP localization: Median nerve/carpal tunnel (89\%); peroneal nerve/fibula head (7.4\%); ulnar nerve/elbow (1.8\%) and tibial nerve/ankle (1.8\%). For associated factors, Gout+Local PN (L-PN) patients were compared with Gout without PN as well as Gout+Systemic PN (S-PN) (see table). Hypertriglyceridemia and dyslipidemia were significantly more frequent among L-PN patients; in other hand, frequency of tophi, STG and mean HAQ values were significantly more frequent in S-PN patients, there were no significant differences among other clinical data associated with gout itself.

Table 1. Factors associated to Gout+PN. Values represent \% unless specified

\begin{tabular}{lcccc}
\hline Variable & $\begin{array}{c}\text { Gout+L-PN } \\
\mathrm{n}=55\end{array}$ & $\begin{array}{c}\text { Gout+S-PN } \\
\mathrm{n}=51\end{array}$ & $\begin{array}{c}\text { Gout Without PN } \\
\mathrm{n}=56\end{array}$ & $\mathrm{p}$ \\
\hline Alcoholism/Smoking & $85 / 69$ & $82 / 68$ & $85 / 73$ & $\mathrm{NS}$ \\
Chronic renal failure & 18 & 17 & 16 & $\mathrm{NS}$ \\
Hyperglycemia/Diabetes & 25 & 31 & 21 & $\mathrm{NS}$ \\
Obesity & 27 & 23 & 29 & $\mathrm{NS}$ \\
Hypertriglyceridemia/Dyslipidemia & $\mathbf{5 8 / 6 9}$ & $27 / 41$ & $35 / 40$ & $\mathbf{0 . 0 2}$ \\
Hypertension & 40 & 28 & 34 & $\mathrm{NS}$ \\
Tophus & 70 & $\mathbf{8 1}$ & 65 & $\mathbf{0 . 0 5}$ \\
STG & 55 & 60 & 41 & $\mathbf{0 . 0 4}$ \\
Index tophi size $(\mathrm{cm})^{\star}$ & $5.3 \pm 3.5$ & $6.5 \pm 4.1$ & $6.2 \pm 4.7$ & $\mathrm{NS}$ \\
HAQ* & $0.38 \pm 0.49$ & $\mathbf{0 . 5 9 \pm 0 . 5 8}$ & $0.37 \pm 0.50$ & $\mathbf{0 . 0 1}$ \\
Serum uric acid* & $8.1 \pm 2.7$ & $7.9 \pm 2.3$ & $7.9 \pm 2.3$ & $\mathrm{NS}$ \\
Previous treatment: & & & $\mathrm{NS}$ & \\
$\quad$ Glucocorticoids & 52 & 68 & 5270 & \\
Colchicine & 51 & 46 & 50 & \\
Allopurinol & 71 & 69 & 70 & \\
\hline
\end{tabular}

\section{*Mean \pm SD.}

Conclusions: PN is common among gout patients, PN could be diagnosed by questionnaires (particularly DN4) and NCS in 65\%. L-PN (median nerve most of them) explain $52 \%$ of the cases, in $48 \%$ S-PN was found, in these group PNP is more frequent. Hypertriglyceridemia is associated with L-PN and STG to S-PN. The role of uric acid and/or crystals needs to be evaluated.

Disclosure of Interest: None declared

DOI: 10.1136/annrheumdis-2017-eular.5060

\section{THU0418 A PROOF-OF-CONCEPT STUDY: TREATING TO THE TARGET WITH URATE LOWERING THERAPY IN REAL-WORLD GOUT PATIENTS}

C.A. Janssen ${ }^{1}$, T.L. Jansen ${ }^{2}$, M.A. Oude Voshaar ${ }^{1}$, H.E. Vonkeman ${ }^{1}$, M.A. van de Laar ${ }^{1} .{ }^{1}$ Arthritis Center Twente, Department of Psychology, Health and Technology, University of Twente, Enschede; ' 2 Department of Rheumatology, Viecuri Medical Center, Venlo, Netherlands

Background: Gouty arthritis is a common, potentially disabling and increasingly prevalent disease [1]. Last year, the European League Against Rheumatism (EULAR) task force gout updated the 2006 recommendations for the management of gout $[2,3]$. The guideline stresses the application of a targeted approach when initiating urate lowering therapy (ULT) in gout patients for reaching the recommended serum urate (sUA) target values. However, data on clinical outcomes of real-world gout patients treated according to this approach are limited.

Objectives: To examine the clinical outcomes achieved in two patient cohorts in which differing targeted ULT treatment approaches were employed, both aiming to reach the EULAR recommended sUA targets.

Methods: We conducted a retrospective chart review study. Gout patients were included that had been treated at the rheumatology departments of two clinical centers in the Netherlands, applying different targeted ULT treatment approaches. Patients in cohort A followed an approach combining two modes of action once allopurinol monotherapy failed to reach the predefined target, whereas patients in cohort B were treated with sequential monotherapy following allopurinol 